\title{
Stability analysis of delay seirepidemic model
}

\author{
Muhammad Altaf Khan ${ }^{1,}{ }^{*}$, Ebenezer Bonyah ${ }^{2}$, Shujaat Ali ${ }^{3}$, Saeed Islam ${ }^{1}$, Saima Naz Khan ${ }^{4}$ \\ ${ }^{1}$ Department of Mathematics, Abdul Wali Khan, University Mardan, Khyber Pakhtunkhwa, Pakistan \\ ${ }^{2}$ Department of Mathematics and Statistics, Kumasi Polytechnic, P. O. Box 854, Kumasi, Ghana \\ ${ }^{3}$ Department of Mathematics, Islamia College University, Peshawar, Khyber Pakhtunkhwa, Pakistan \\ ${ }^{4}$ Department of Physics, Abdul Wali Khan University Mardan, Khyber Pakhtunkhwa, Pakistan
}

\section{A R T I C L E I N F O}

\section{Article history:}

Received 27 May 2016

Received in revised form

21 July 2016

Accepted 21 July 2016

\section{Keywords:}

SEIR epidemic model

Reproduction number

Global stability

Numerical results

\section{Introduction}

In mathematical epidemiology, the constructing and study of models that describe the disease information about the spread and control the infectious disease is the most important major research area of biology. The first who introduced the model is called the "SIR", S-I-R (susceptible, infected, recovered) in 1927 by Kermack and McKendrick (1927). They described a simple SIR model in a closed population over time and incorporated the theoretical number of infected individuals with a contagious disease. The dynamic process between the susceptible and infected individuals is the disease transmission. The analysis and behavior of the SIR type models is greatly influenced by which the transmission among the infective individuals and susceptible are modeled. Often, the epidemiological models are based on the so-called mass action (Anderson et al., 1991). The models, in which realistic transmission functions are used by authors have faced some problems but consequently gained much attention (Ruan and Wang, 2003; Xiao and Ruan, 2007).

Study of mathematical models incorporating the exposed class plays important role in epidemiology due to importance of incubation period of such diseases, like Dengue fever (3 to 14) days. In

* Corresponding Author.

Email Addresses: altafdir@gmail.com (M. Khan), shujaatuom21@gmail.com (S. Ali), saeedislam@awkum.edu.pk (S. Islam)

http://dx.doi.org/10.21833/ijaas.2016.07.008

2313-626X/C) 2016 The Authors. Published by IASE.

This is an open access article under the CC BY-NC-ND license

(http://creativecommons.org/licenses/by-nc-nd/4.0/) epidemiology the incubation period or latency can be modeled by adding the exposed class to the model to understand its behavior and dynamics (Gubler, 1988). In Gubler (1988), the delay SIR and delay SEIR models and the comparison for the reproduction number were presented. In literature, the delay differential equations have been used for a variety of models such as, SIR, SEIR, SIS and SIRS. An S-I-S epidemic model with the constant time delay has been studied by Hethcote and Van den Driessche (1995), admitting the duration of infectiousness.

Beretta et al. (2001) studied the global stability in SIR epidemic model with distributed delay which described the time taking for an individual to lose infection. Further, with the effect of time delay on the stability of an endemic equilibrium has been studied by Song and Cheng (2005). They provided the conditions in which the stability of endemic equilibrium exists for all delays. Mathematical models that describe epidemiology are widely used for the analysis of global stability of infection free and endemic equilibrium. Many authors worked on the infectious diseases such as Berezovsky et al. (2005), Esteva and Matias, (2001) Greenhalgh (1992), Hsu and Zee (2014), Yi et al. (2009), Zhang et al. (2008) and the references therein.

In this work, we study an epidemic SEIR model with the information variable. The work of Kar and Mondal (2011) motivated us for current study, and incorporated the $E(t)$ (exposed Class) to our new model, and studying the model with the new information variable. By incorporating this class, the analysis of SEIR model with information variable will be more interesting for readers and researchers. 
The structure of the paper is as follow: We formulate the model in Section 2. In Section 3, we present the local stability for the model. In Section 4, we find the global stability of the endemic equilibrium. The numerical solution of the model is presented in Section 5. Finally, discussion with conclusion is presented in Section 6.

\section{Model constructions}

We consider a mathematical model of the type (Eq. 1):

$$
\begin{aligned}
& \frac{d S}{d t}=\Gamma S\left(1-\frac{S}{k}\right)-\frac{\beta S I}{1+a S}, \\
& \frac{d E}{d t}=\frac{\beta S I}{1+a S}-\mu E-\mu_{1} E, \\
& \frac{d I}{d t}=\mu_{1} E-\mu_{2} I-\mu I, \\
& \frac{d R}{d t}=\mu_{2} I-\mu R,
\end{aligned}
$$

with initial conditions (Eq. 2):

$$
\begin{aligned}
& S(0)=S_{o} \geq 0, \quad E(0)=E_{o} \geq 0, \quad I(0)=I_{o} \geq \\
& 0, \quad R(0)=R_{o} \geq 0 .
\end{aligned}
$$

Here, $S(t)$ is the density of susceptible within the population, $E(t)$ is the density of exposed individuals $I(t)$ is the infected and $R(t)$ is the density of recovered. $\mu$ shows the natural death rate of the population, $\beta$ represents the disease contact rate and $\Gamma$ is the intrinsic growth rate. The exposed individuals infected at a rate of $\mu_{1}$ and move to the class of the infected. The recovery rate from infection is shown by $\mu_{2}$ and $k$ is the carrying capacity of the susceptible. The saturation term which measures the inhibitory effect is shown by $a$. Here, we use the new variable $Z$, known to be the information variable that collects information for the disease present state, i.e. depending on present values and some previous values of the state variables. Many authors used this variable in their model such as D'Onofrio et al. (2007), D'Onofrio, Manfredi (2007), Buonomo et al. (2008), Kar and Mondal (2011). We take the formula (Eq. 3):

$$
Z(t)=\int_{-\infty}^{t} f(S(\tau), I(\tau)) K(t-\tau) d \tau
$$

here $K(t-\tau)$ represents the delaying kernel (Wang et al., 2014), the distributed delay is $\tau$, that shows for any time $t$ the susceptible, $S$, the exposed people, $\mathrm{E}$, and infected people, I can be affected with these variables $\mathrm{S}, \mathrm{E}$ and I, possibly, at some previous times $\tau \leq \tau$. In this work, we assume $g(S, I)=S$, and $K(t-\tau)=\frac{1}{T} \exp ^{-\frac{1}{T}(t-\tau)}$, where $\mathrm{T}$ shows the average delay of the summarized information and also concerning the historical memory on the concern disease. With these assumptions, the following system is presented (Eq. 4):

$$
\begin{aligned}
& \frac{d S}{d t}=\Gamma S\left(1-\frac{S}{k}\right)-\frac{\beta S I}{1+a S}, \\
& \frac{d E}{d t}=\frac{\beta I Z}{1+a Z}-\mu E-\mu_{1} E, \\
& \frac{d I}{d t}=\mu_{1} E-\mu_{2} I-\mu I, \\
& Z(t)=\int_{-\infty}^{t} f(S(\tau), I(\tau)) K(t-\tau) d \tau, \\
& \frac{d R}{d t}=\mu_{2} I-\mu R,
\end{aligned}
$$

the system (4) is in the form of the non-linear Integro-differential system. By transformation, we can write it in the form of non-linear ordinary differential equations (Eq. 5):

$$
\begin{aligned}
& \frac{d S}{d t}=\Gamma S\left(1-\frac{S}{k}\right)-\frac{\beta S I}{1+a S}, \\
& \frac{d E}{d t}=\frac{\beta I Z}{1+a Z}-\mu E-\mu_{1} E, \\
& \frac{d I}{d t}=\mu_{1} E-\mu_{2} I-\mu I, \\
& \frac{d Z}{d t}=\frac{1}{T}(S-Z), \\
& \frac{d R}{d t}=\mu_{2} I-\mu R,
\end{aligned}
$$

in system (5), the last equation is independent of the rest, so we omit it, because $\mathrm{R}$ depends only on I. So the model becomes (Eq. 6):

$$
\begin{aligned}
& \frac{d S}{d t}=\Gamma S\left(1-\frac{S}{k}\right)-\frac{\beta S I}{1+a S}, \\
& \frac{d E}{d t}=\frac{\beta I Z}{1+a Z}-\mu E-\mu_{1} E, \\
& \frac{d I}{d t}=\mu_{1} E-\mu_{2} I-\mu I, \\
& \frac{d Z}{d t}=\frac{1}{T}(S-Z),
\end{aligned}
$$

where

$$
\Gamma, \beta, k, a, \mu, \mu_{2}, T>0 \text {. }
$$

\section{Stability analysis}

In this section, we present the local stability analysis of the system (6). In the subsection (3.1), we present the local stability of disease free and the subsequent section (3.2) we find the local stability of endemic equilibrium.

\subsection{Disease free stability}

The disease free equilibrium point of system (6) is (Eq. 7).

Theorem: An unstable equilibrium exists for All $T>0$ at $\bar{E}_{0}$.

Proof: At the equilibrium point $\bar{E}_{0}$, the Jacobean matrix is (Eq. 8).

The eigenvalues associated to $J_{0}(0,0,0,0)$ are, $\Gamma,-\left(\mu+\mu_{1}\right),-\left(\mu+\mu_{2}\right)$ and $-\frac{1}{T}$. This proves the instability of the system at $\bar{E}_{1}$.

Theorem: If $R_{0}<1$, the jacobian matrix at $\bar{E}_{1}$ is locally asymptotically stable for all $\mathrm{T}>0$. 
Proof: The jacobian matrix at $\bar{E}_{1}$ is (Eq. 9). The eigenvalues associated to Jacobian matrix at $\bar{E}_{1}$ are, $-\Gamma,-\frac{1}{T},-\left(\mu+\mu_{1}\right)$ and $-\left(\mu+\mu_{2}\right)$.
Theorem: (i) The model (6), at $\overline{E_{0}}=(0,0,0,0)$ has a trivial equilibrium point while at $\bar{E}_{1}=(k, 0,0, k)$ the disease free equilibrium exists.

$$
\begin{aligned}
& J_{0}(S, E, I, Z)=\left(\begin{array}{l}
\Gamma\left(1-\frac{S}{k}\right)-\frac{\beta I}{1+a S}+S\left(-\frac{\Gamma}{k}+\frac{\alpha \beta I}{(1+a S)^{2}}\right) \\
0 \\
0 \\
\frac{1}{T}
\end{array}\right. \\
& J_{0}(0,0,0,0)=\left(\begin{array}{llll}
\Gamma & 0 & 0 & 0 \\
0 & -\left(\mu+\mu_{1}\right) & 0 & 0 \\
0 & \mu_{1} & -\left(\mu+\mu_{2}\right) & 0 \\
\frac{1}{T} & 0 & 0 & -\frac{1}{T}
\end{array}\right) \\
& J_{0}(k, 0,0, k)= \\
& \left(\begin{array}{llll}
-\Gamma & 0 & -\frac{\beta k}{1+a k} & 0 \\
0 & -\left(\mu+\mu_{1}\right) & 0 & 0 \\
0 & \mu_{1} & -\left(\mu+\mu_{2}\right) & 0 \\
\frac{1}{T} & 0 & 0 & -\frac{1}{T}
\end{array}\right)
\end{aligned}
$$

(ii) Let $R_{0}>1$, for system (6), a unique positive endemic equilibrium exists at:

$$
\overline{E_{2}}=\left(S^{*}, E^{*}, I^{*}, Z^{*}\right),
$$

when $\left(a\left(\mu+\mu_{2}\right)>\mu_{1} \beta\right)$ where $R_{0}=\frac{\left(\left(\mu+\mu_{2}\right)+\mu_{1} \beta\right)}{a\left(\mu+\mu_{2}\right)}$ is the basic reproduction number and

$$
\begin{aligned}
& S^{*}=Z^{*}=\frac{\left(\mu+\mu_{2}\right)}{\left(a\left(\mu+\mu_{2}\right)-\mu_{1} \beta\right)}, \\
& E^{*}=\frac{a\left(\mu+\mu_{2}\right)^{2}}{\mu_{1} \beta^{2}}\left(R_{0}-1\right), \\
& I^{*}=\frac{a \mu\left(\mu+\mu_{2}\right)}{\mu_{1} \beta^{2}}\left(R_{0}-1\right), \\
& R^{*}=\frac{a \mu_{2}\left(\mu+\mu_{2}\right)}{\mu_{1} \beta^{2}}\left(R_{0}-1\right) .
\end{aligned}
$$

$$
\left.\begin{array}{lll}
0 & -\frac{\beta S}{1+a S} & 0 \\
-\left(\mu+\mu_{1}\right) & \frac{\beta Z}{1+a Z} & \frac{\beta I}{(1+a Z)^{2}} \\
\mu_{1} & -\left(\mu+\mu_{2}\right) & 0 \\
0 & 0 & -\frac{1}{T}
\end{array}\right)
$$

Proof: (i): The first is easy to prove. (ii): The proof of the theorem is easy, when $R_{0}>1$, clearly, shows the unique endemic equilibrium point.

\subsection{Local stability of endemic equilibrium}

In this subsection, we present the local stability of the system (6) at the endemic equilibrium point $\bar{E}_{2}$. In order to do this, in the following we state and prove the results.

Theorem: The endemic equilibrium point $\bar{E}_{2}$, of the system (6) is stable locally asymptotically if $R_{0}>1$, provided that $\left(2 \mu\left(\mu+\mu_{2}\right) \geq \mu a k\left(\mu+\mu_{2}\right)\right)$ and $\left(\frac{\mu_{3}}{\beta}>\mu\right)$ are satisfied.

Proof: The Jacobian matrix of the system (6), at the endemic equilibrium point $\bar{E}_{2}$ is given by:

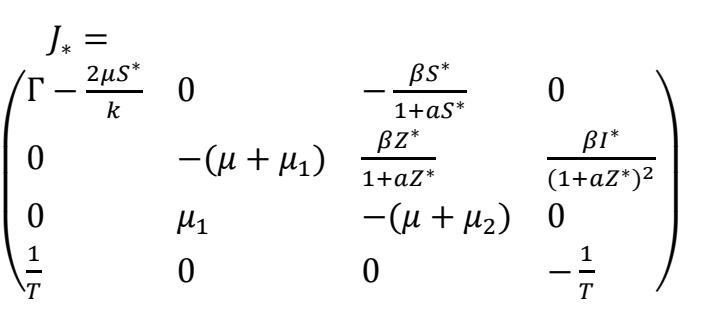

The Jacobian matrix $J_{*}$ gives the following equation (Eq. 10),

$$
\lambda^{4}+a_{1} \lambda^{3}+a_{2} \lambda^{2}+a_{3} \lambda+a_{4}=0,
$$

where

$$
\begin{gathered}
a_{1}=\frac{1}{T}-\Gamma+\frac{2(k+S) \mu}{k}+\mu_{1}+\mu_{2}, \\
a_{2}=\frac{\mu+\mu_{1}}{T}+\frac{S \beta \mu_{1}}{1+a S}+\frac{\mu+\mu_{2}}{T}+\left(\mu+\mu_{1}\right)\left(\mu+\mu_{2}\right)-\left(\Gamma-\frac{2 S \mu}{k}\right)\left(\left(\mu+\mu_{1}\right)+\left(\mu+\mu_{2}\right)+\frac{1}{T}\right), \\
a_{3}=-\frac{S \beta \mu_{1}}{T+a S T}+\frac{S \beta\left(\Gamma-\frac{2 S \mu}{k}\right) \mu_{1}}{1+a S}-\frac{(k \Gamma-2 S \mu)\left(\mu+\mu_{1}\right)}{k T}-\frac{(k \Gamma-2 S \mu)\left(\mu+\mu_{2}\right)}{k T}+\frac{\left(\mu+\mu_{1}\right)\left(\mu+\mu_{2}\right)}{T} \\
-\frac{(k \Gamma-2 S \mu)\left(\mu+\mu_{1}\right)\left(\mu+\mu_{2}\right)}{k}, \\
a_{4}=\left(\Gamma-\frac{2 S \mu}{k}\right)\left(\frac{\left(\mu+\mu_{1}\right)\left(\mu+\mu_{2}\right)}{T}+\frac{S \beta \mu_{1}}{(1+a S) T}\right)+\frac{S I \beta^{2} \mu_{1}}{(1+a S)^{3} T} .
\end{gathered}
$$

where

$$
Q_{1}=\left(\mu+\mu_{1}\right), Q_{2}=\left(\mu+\mu_{2}\right) .
$$

The Routh-Hurtwiz conditions are satisfied with some sufficient conditions. So, the local stability of 
the endemic equilibrium point $\bar{E}_{2}$ of the system (6) is locally asymptotically stable.

\section{Global stability}

In this section, we show the global stability for the system (6). We use the method presented in ( $\mathrm{Li}$ et al., 1996), to obtain the global stability for our model. The disease persistence occurs when the endemic equilibrium is stable globally asymptotically. In such a case, the disease permanently exists in the community. Here, we consider the subsystem of (6),

$$
\begin{aligned}
& \frac{d S}{d t}=\Gamma S\left(1-\frac{S}{k}\right)-\frac{\beta S I}{1+a S}, \\
& \frac{d \mathrm{E}}{\mathrm{dt}}=\frac{\beta \mathrm{IZ}}{1+\mathrm{aZ}}-\mu \mathrm{E}-\mu_{1} \mathrm{E}, \\
& \frac{\mathrm{dI}}{\mathrm{dt}}=\mu_{1} \mathrm{E}-\mu_{2} \mathrm{I}-\mu \mathrm{I},
\end{aligned}
$$

Theorem: For $\mathrm{R}_{0}>1$, the endemic equilibrium point $\bar{E}_{2}$, of the subsystem (11) is stable global asymptotically.

Proof: The second additive compound matrix $J_{*}$, of subsystem (11) is (Eq. 12):

$$
\mathrm{J}_{*}=\left(\begin{array}{lll}
\Gamma-\frac{2 \mu \mathrm{S}^{*}}{\mathrm{k}} & 0 & -\frac{\beta \mathrm{S}^{*}}{1+\mathrm{aS} \mathrm{S}^{*}} \\
0 & -\left(\mu+\mu_{1}\right) & \frac{\beta \mathrm{Z}^{*}}{1+\mathrm{aZ}} \\
0 & \mu_{1} & -\left(\mu+\mu_{2}\right)
\end{array}\right)
$$

consider the function:

$$
P=P(S, E, I)=\operatorname{diag}\left(\frac{S}{E}, \frac{S}{E}, \frac{S}{E}\right),
$$

then

$$
\begin{aligned}
& \mathrm{P}^{-1}=\operatorname{diag}\left(\frac{\mathrm{E}}{\mathrm{S}}, \frac{\mathrm{E}}{\mathrm{S}}, \frac{\mathrm{E}}{\mathrm{S}}\right) \\
& \mathrm{P}_{\mathrm{f}}=\operatorname{diag}\left(\frac{\mathrm{ES} \prime-\mathrm{SE}^{\prime}}{\mathrm{E}^{2}},\left|\frac{\mathrm{ES} \prime-\mathrm{SE} \prime}{\mathrm{E}^{2}},\right| \frac{\mathrm{ES} \prime-\mathrm{SE} \prime}{\mathrm{E}^{2}}\right),
\end{aligned}
$$

And

$$
\begin{aligned}
& \mathrm{P}_{\mathrm{f}} \mathrm{P}^{-1}=\left(\frac{\mathrm{S}^{\prime}}{\mathrm{S}}-\frac{\mathrm{E}^{\prime}}{\mathrm{E}}, \frac{\mathrm{S}^{\prime}}{\mathrm{S}}-\frac{\mathrm{E}^{\prime}}{\mathrm{E}}, \frac{\mathrm{S}^{\prime}}{\mathrm{S}}-\frac{\mathrm{E}^{\prime}}{\mathrm{E}}\right), \\
& \text { also, } \mathrm{J}^{*}=\mathrm{PJ}^{*} \mathrm{P}^{-1} . \\
& \mathrm{B}=\mathrm{P}_{\mathrm{f}} \mathrm{P}^{-1}+\mathrm{PJ}^{*} \mathrm{P}^{-1}=\left(\begin{array}{ll}
\mathrm{B}_{11} & \mathrm{~B}_{12} \\
\mathrm{~B}_{21} & \mathrm{~B}_{22}
\end{array}\right)
\end{aligned}
$$

where

$$
\begin{aligned}
& \mathrm{B}_{11}=\frac{\mathrm{S} \prime}{\mathrm{S}}-\frac{\mathrm{E}^{\prime}}{\mathrm{E}}+\Gamma-\frac{2 \mu \mathrm{S}}{\mathrm{k}}, \mathrm{B}_{12}=\left(0-\frac{\beta \mathrm{S}}{1+\mathrm{aS}}\right), \mathrm{B}_{21}= \\
& \left(\begin{array}{ll}
0 & 0
\end{array}\right)^{\mathrm{T}}, \\
& B_{22}=\left[\begin{array}{cc}
\frac{S^{\prime}}{S}-\frac{E^{\prime}}{E}-\left(\mu+\mu_{1}\right) & \frac{\beta Z}{1+a Z} \\
\mu_{1} & \frac{S^{\prime}}{S}-\frac{E^{\prime}}{E}-\left(\mu+\mu_{2}\right)
\end{array}\right]
\end{aligned}
$$

consider the norm in $\mathrm{R}^{3}$ as: $|(\underline{\mathrm{u}}, \underline{\mathrm{v}}, \underline{\mathrm{w}})|=\max (|\underline{\mathrm{u}}|,|\underline{\mathrm{v}}|,|\underline{\mathrm{w}}|)$

where $(\underline{\mathrm{u}}, \underline{\mathrm{v}}, \underline{\mathrm{w}})$, represents the vector in $\mathrm{R}^{3}$ and shows by $\ell$ the Lozinskii measure w. r. to the norm in (Martin, 1974).

$$
\begin{aligned}
& \ell(B) \leq \sup \left\{g_{1}, g_{2}\right\} \\
& =\sup \left\{\ell\left(B_{11}\right)+\left|B_{12}\right|, \ell\left(B_{22}\right)+\left|B_{21}\right|\right\},
\end{aligned}
$$

here, $\left|B_{12}\right|,\left|B_{21}\right|$ represents the matrix norm with respect to $\mathrm{L}^{1}$ vector norm and $\ell_{1}$ denote the Lozinskii measure with respect to the $\mathrm{L}^{1}$ norm ${ }^{1}$.

$$
\begin{aligned}
& \ell\left(B_{11}\right)=\frac{S \prime}{S}-\frac{E^{\prime}}{E}+\Gamma-\frac{2 \mu S}{k}, \\
& B_{12}=\left|\frac{\beta S}{1+a S}\right|
\end{aligned}
$$

and using $\frac{E \prime}{E}=\frac{\beta Z}{(1+a Z) E}-\mu-\mu_{1}$, and second equation of system (6) we get (Eq. 12-16):

$\therefore \mathrm{g}_{1}=\ell\left(\mathrm{B}_{11}\right)+\left|\mathrm{B}_{12}\right|$,

$=\frac{\mathrm{S} \prime}{\mathrm{S}}-\frac{\mathrm{E} \prime}{\mathrm{E}}+\Gamma-\frac{2 \mu \mathrm{S}}{\mathrm{k}}+\frac{\beta \mathrm{S}}{1+\mathrm{aS}}$,

$=\frac{\mathrm{S} \prime}{\mathrm{S}}-\frac{\beta \mathrm{IZ}}{(1+\mathrm{aZ}) \mathrm{E}}+\mu+\mu_{1}+\Gamma-2 \mu \frac{\mathrm{S}}{\mathrm{k}}+\frac{\beta \mathrm{S}}{1+\mathrm{aS}}$,

$=\frac{\mathrm{S} \prime}{\mathrm{S}}+\mu_{1}+\max \left\{\mu-\frac{\beta \mathrm{IZ}}{(1+\mathrm{aZ}) \mathrm{E}}+\Gamma-2 \mu \frac{\mathrm{S}}{\mathrm{k}}+\frac{\beta \mathrm{S}}{1+\mathrm{aS}}\right\}$

$\therefore \mathrm{g}_{2}=\ell\left(\mathrm{B}_{22}\right)+\left|\mathrm{B}_{21}\right|$,

$=\frac{S^{\prime}}{S}-\frac{E^{\prime}}{E}-\left(\mu+\mu_{1}\right)-\left(\mu+\mu_{2}\right)+\mu_{1}+\frac{\beta Z}{1+a Z}$,

$=\frac{\mathrm{S}^{\prime}}{\mathrm{S}}-\frac{\beta \mathrm{IZ}}{(1+\mathrm{aZ}) \mathrm{E}}-\left(\mu+\mu_{2}\right)+\mu_{1}+\frac{\beta \mathrm{Z}}{1+\mathrm{aZ}}$,

$=\frac{S^{\prime}}{S}+\mu_{1}+\max \left\{\frac{\beta Z}{1+a Z}-\left(\mu+\mu_{2}\right)+\frac{\beta I Z}{(1+a Z) E}\right.$,

$\left.2 \mu \frac{\mathrm{S}}{\mathrm{k}}-\Gamma-\mu-\frac{\beta \mathrm{S}}{1+\mathrm{aS}}+\frac{\beta \mathrm{IZ}}{(1+\mathrm{aZ}) \mathrm{E}}\right\}$

i. e.

$$
\begin{aligned}
& \ell(B)=\frac{S^{\prime}}{\mathrm{s}}+\mu_{1}-\min \left\{-\frac{\beta \mathrm{Z}}{1+\mathrm{aZ}}+\left(\mu+\mu_{2}\right)+\frac{\beta \mathrm{IZ}}{(1+\mathrm{aZ}) \mathrm{E}},\right. \\
& \frac{\beta \mathrm{IZ}}{(1+\mathrm{aZ}) \mathrm{E}}+2 \mu \frac{\mathrm{s}}{\mathrm{k}}-\Gamma-\mu- \\
& \left.\frac{\beta \mathrm{S}}{1+\mathrm{as}}\right\} \quad \begin{array}{l}
\quad(15)(\mathrm{B}) \leq \frac{\mathrm{s}}{\mathrm{s}}+\mu-\omega, \quad \omega>0, \\
=\frac{\mathrm{s} \prime}{\mathrm{s}}-(\omega-\mu),
\end{array}
\end{aligned}
$$$$
\text { i. e. }
$$$$
\frac{1}{t} \int_{0}^{t} \ell(B) d S \leq \frac{1}{t} \log \frac{S(t)}{S(0)}-(\omega-\mu),
$$

i. e.

$$
\limsup _{t \rightarrow \infty} \sup \frac{1}{t} \int_{0}^{t} \ell(B) d S \leq-(\omega-\mu)<0 .
$$

Now, consider the limit system,

$$
\frac{\mathrm{dZ}_{*}}{\mathrm{dt}}=\frac{1}{\mathrm{~T}}\left(\mathrm{~S}_{*}-\mathrm{Z}_{*}\right)
$$

based on (19),

$$
\mathrm{Z}(\mathrm{t})=\mathrm{e}^{-\frac{\mathrm{t}}{\mathrm{T}}}\left[\mathrm{Z}(0)+\frac{\mathrm{S}_{*}}{\mathrm{~T}} \int_{0}^{\mathrm{t}} \mathrm{e}^{\frac{\mathrm{s}}{\mathrm{T}}} \mathrm{ds}\right]
$$


which implies that

$$
\mathrm{Z}(\mathrm{t}) \rightarrow \frac{\mathrm{S}_{*}}{\mathrm{~T}}=\mathrm{Z}_{*}, \quad \text { as } \mathrm{t} \rightarrow \infty
$$

then, we get that $\mathrm{E}^{*}$ is globally asymptotically stable. The proof is completed.

\section{Numerical results}

In this section, we find the numerical solution of the proposed model (6). For the values, $\Gamma=2, \mathrm{k}=$ $5.7, \beta=0.5, \mathrm{a}=0.01, \mu_{1}=0.4, \mu_{2}=0.2, \mu=0.2$, the positive equilibrium of the system at $\overline{\mathrm{E}}_{2}$ is $\overline{\mathrm{E}}_{2}=(2.45902,4.6606,2.3303,2.45902)$. The

eigenvalues of the system $\bar{E}_{1}$ are $(-2 .,-1.72414,-0.4$,$0.2)$.

\section{Discussion and conclusion}

We have successfully presented and analyzed a delay SEIR epidemic (special type) model that contains the information variable, Depending on the present values of the variables of the state. We have analyzed the model and found its basic reproduction number $R_{0}$. We observed that when $R_{0}<1$, then disease free equilibrium is stable locally asymptotically and the disease comes to halt in the community and an unstable endemic equilibrium exists when $R_{0}>1$, the disease becomes endemic and will spread in the community. Further, we observed that the value of $R_{0}$ at $E_{0}=(0,0,0,0)$ the model is unstable and when $\mathrm{E}_{1}=(1,0,0,0)$ the model becomes stable. The delay parameter $T$ also affects the endemic equilibrium. The endemic equilibrium is locally stable when, $\mathrm{R}_{0}>1$, together with some conditions. For $\mathrm{R}_{0}>1$, we observed that the model is globally asymptotically stable. For the value of $\mathrm{T}=0.38$, the endemic equilibrium is stable locally asymptotically and unstable equilibrium exists for the value of $\mathrm{T}>0.38$. Moreover, we observed that when $\mathrm{T}=0.58$, the endemic equilibrium becomes unstable.

Finally, we have presented the numerical solutions of the model and the theoretical results are justified in the form of Figs. 1 to 4 . Fig. 1 is the result of the basic reproduction number $\mathrm{R}_{0}$, when $\mathrm{R}_{0}<1$, a stable disease free equilibrium exists and when $\mathrm{R}_{0}>1$, and unstable endemic equilibrium exist. Fig $2-4$, shows the behavior of the model for the suggested values. In Fig. 2, a stable equilibrium exists. In Fig. 3, a stable equilibrium exists only for the case when $\Gamma=6$ and $\mathrm{T}=0.68$. An unstable equilibrium exists when $\mathrm{T}>0.38$. Figs. $5-7$ show the phase portrait of the model (6).

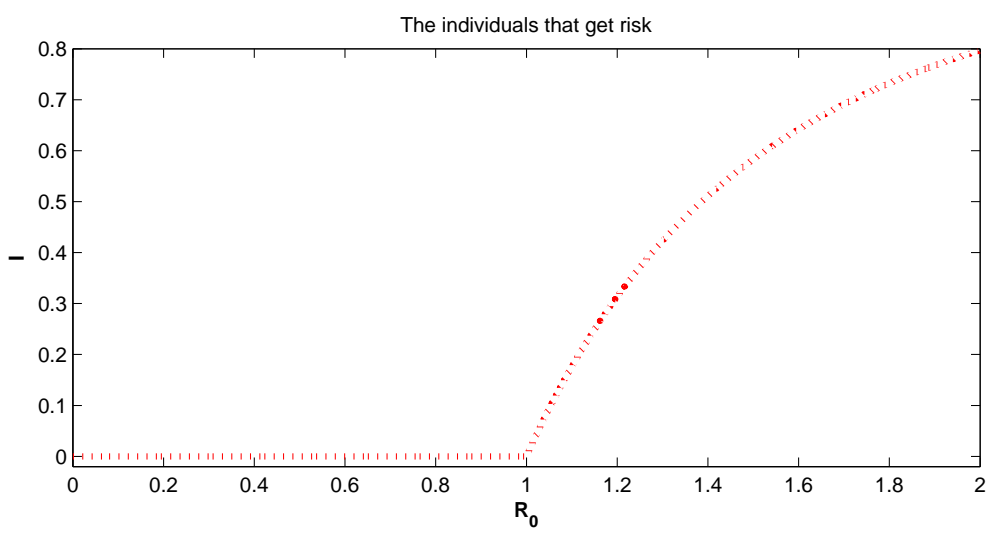

Fig. 1: When $R_{0}>1$, the individuals get risk

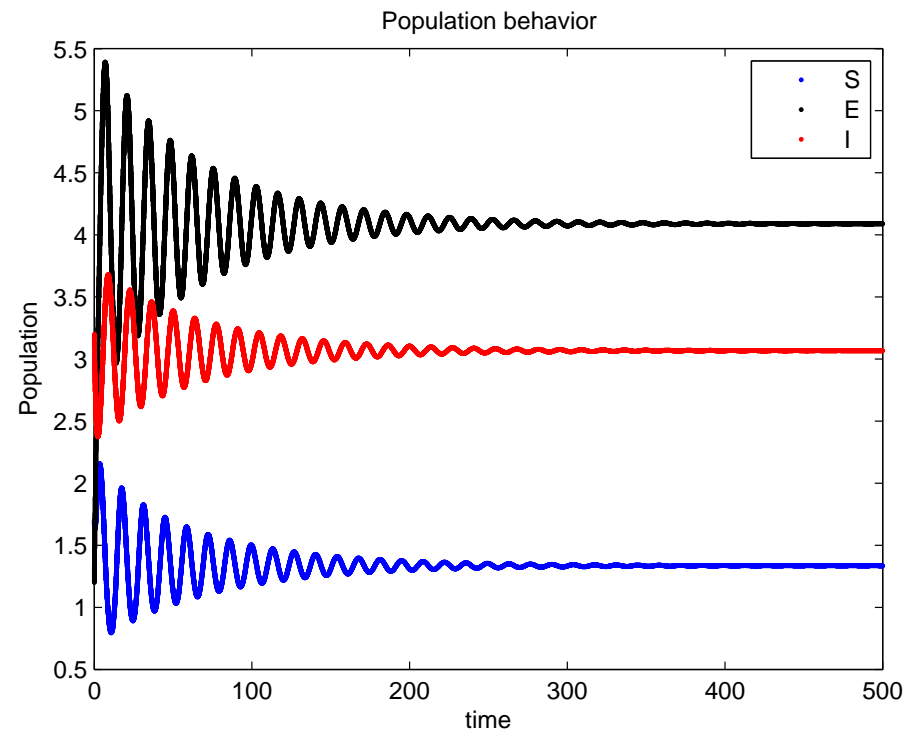

Fig. 2: Plot with parameter values $\Gamma=2, \mathrm{k}=5.7, \beta=0.5, \alpha=0.001, \mu_{1}=0.4, \mu_{1}=0.2, \mathrm{~T}=0.38, \mu=0.2$ 


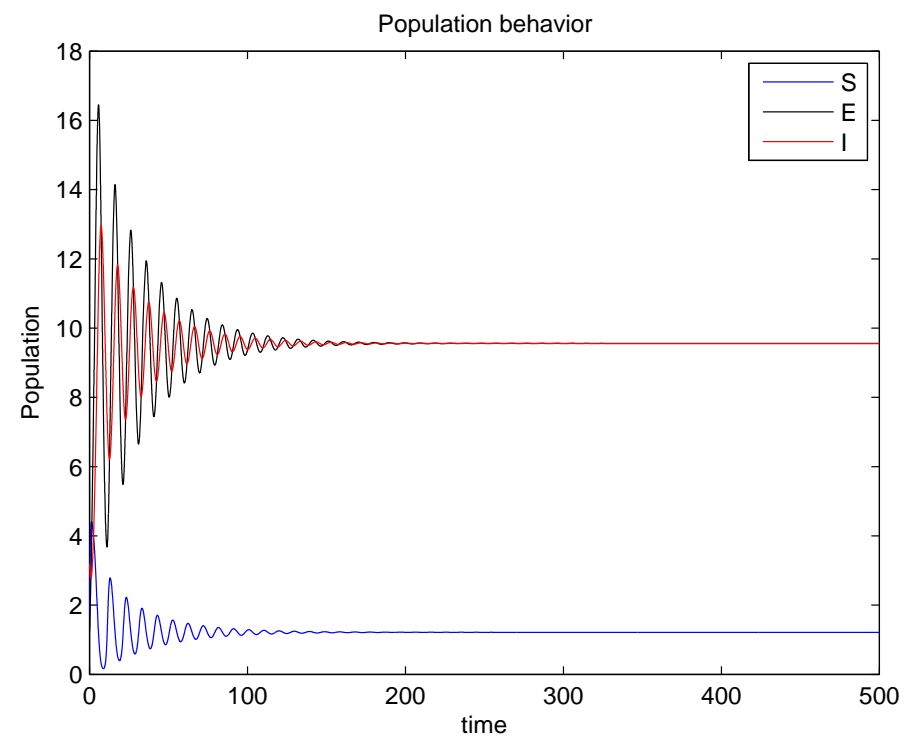

Fig. 3: Stable equilibrium exists when $\Gamma=6$ and $T=0.68$ and taking the rest of the parameters are same

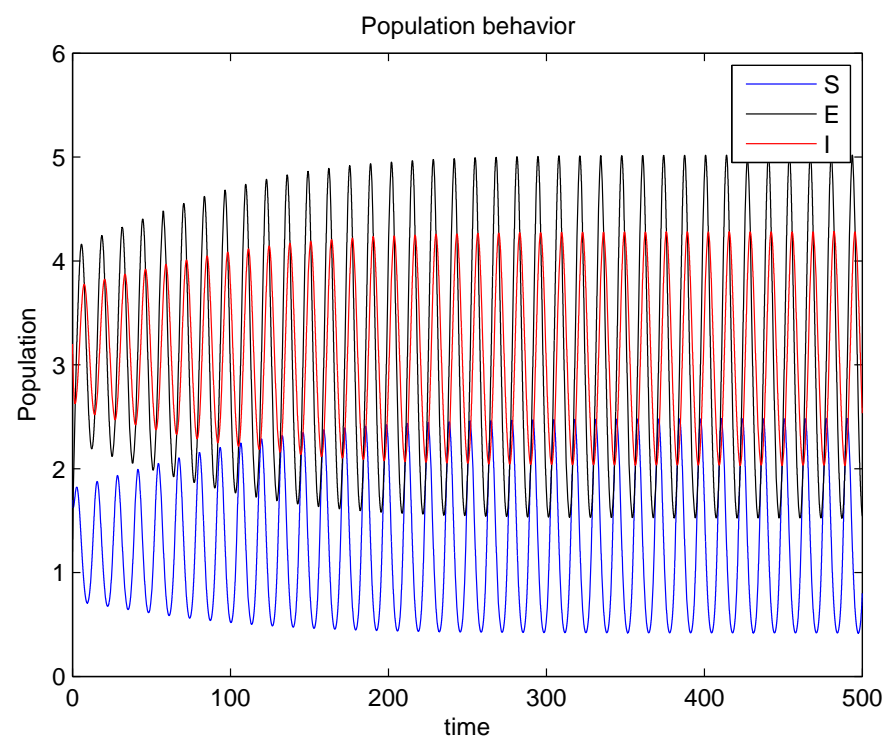

Fig. 4: Unstable positive equilibrium of system (6), when $T=0.58$

(a)

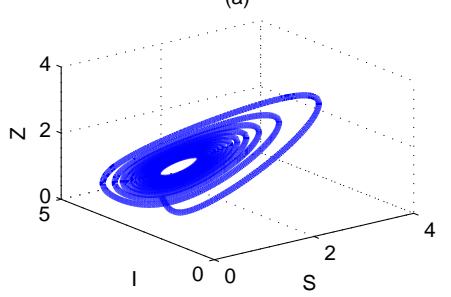

(b)
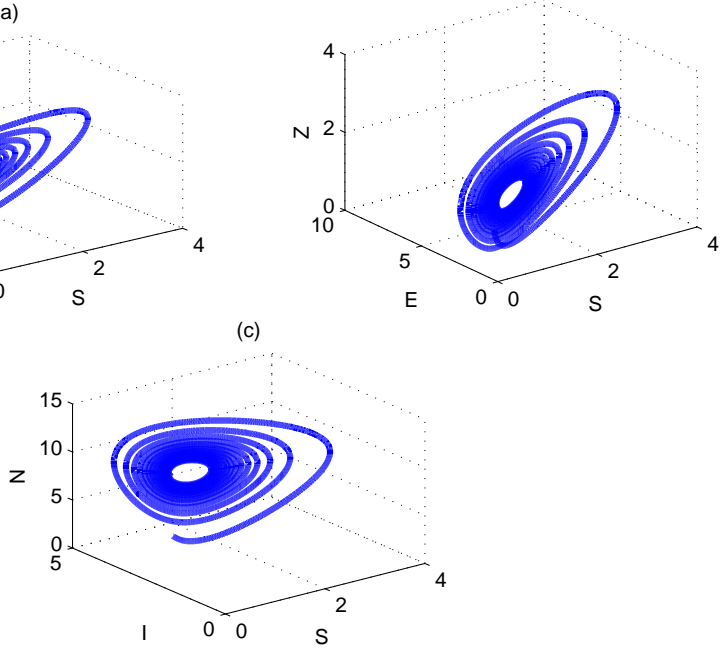

Fig. 5: Phase portrait of the proposed model (6), when $T=0.38$ 


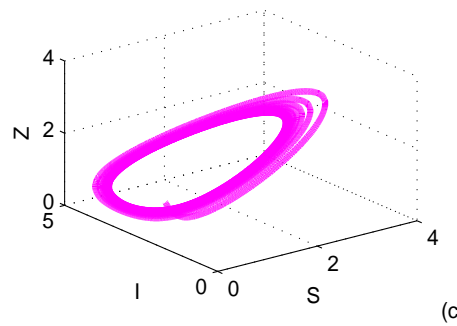

(b)

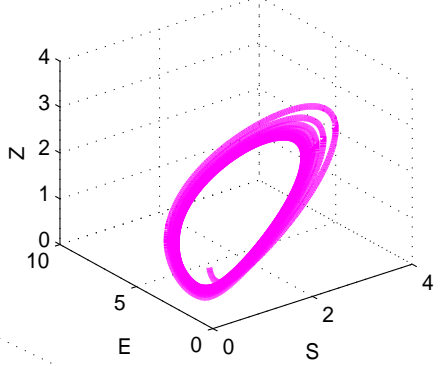

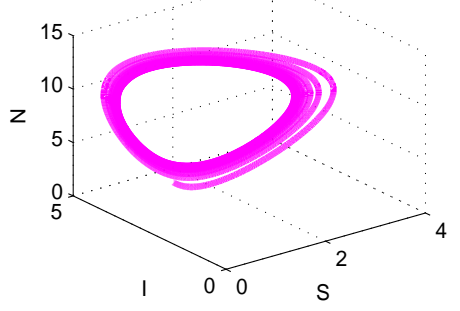

Fig. 6: Phase portrait of the proposed model (6), when $T=0.58$

(a)

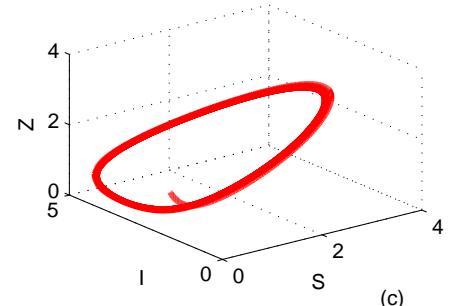

(b)

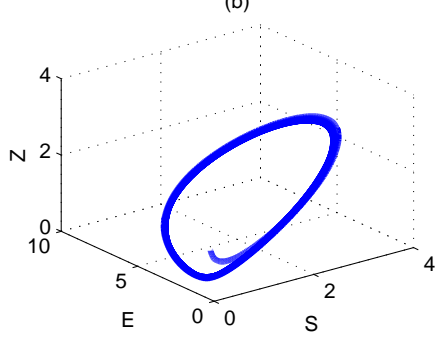

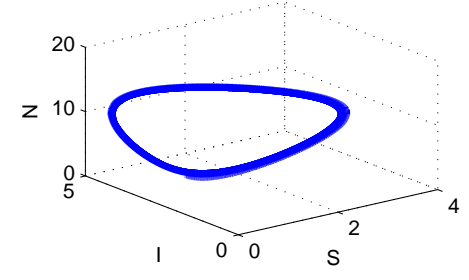

Fig. 7: Phase portrait of the proposed model (6), when $T=0.68$

\section{Conflict of Interests}

The authors have declared that no competing interests exist.

\section{Source of Funding}

No current funding sources for this study.

\section{References}

Anderson RM, May RM and Anderson B (1992). Infectious diseases of humans: dynamics and control. Oxford University Press, Oxford: 28.

Beretta E, Hara T, Ma W and Takeuchi Y (2001). Global asymptotic stability of an SIR epidemic model with distributed time delay. Nonlinear Analysis: Theory, Methods and Applications, 47(6): 4107-4115.

Berezovsky F, Karev G, Song B and Castillo-Chavez C (2005). A simple epidemic model with surprising dynamics. Mathematical Biosciences and Engineering, 2(1): 133-152.
Buonomo B, d'Onofrio A and Lacitignola D (2008). Global stability of an SIR epidemic model with information dependent vaccination. Mathematical Biosciences, 216(1): 9-16.

d'Onofrio A, Manfredi P and Salinelli E (2007). Vaccinating behaviour, information, and the dynamics of SIR vaccine preventable diseases. Theoretical Population Biology, 71(3): 301-317.

d'Onofrio A and Manfredi P (2007). Bifurcation thresholds in an SIR model with informationdependent vaccination. Mathematical Modelling of Natural Phenomena, 2(1): 26-43.

Esteva L and Matias M (2001). A model for vector transmitted diseases with saturation incidence. Journal of Biological Systems, 9(4): 235-245.

Greenhalgh D (1992). Some results for an SEIR epidemic model with density dependence in the death rate. Mathematical Medicine and Biology, 9(2): 67-106. 
Gubler DJ (1998). Dengue and dengue hemorrhagic fever. Clinical Microbiology Reviews, 11(3): 480496.

Hethcote HW and Van den Driessche P (1995). An SIS epidemic model with variable population size and a delay. Journal of Mathematical Biology, 34(2): 177-194.

Hsu S and Zee A (2004). Global spread of infectious diseases. Journal of Biological Systems, 12(3): 289-300.

Kar TK and Mondal PK (2011). Global dynamics and bifurcation in delayed SIR epidemic model. Nonlinear Analysis: Real World Applications, 12(4): 2058-2068.

Kermack WO and McKendrick AG (1927). A contribution to the mathematical theory of epidemics. In Proceedings of the Royal Society of London A: Mathematical, Physical and Engineering Sciences, The Royal Society, 115(772):700-721.

Li MY and Muldowney JS (1996). A geometric approach to global-stability problems. SIAM Journal on Mathematical Analysis, 27(4): 10701083.

Martin RH (1974). Logarithmic norms and projections applied to linear differential systems.
Journal of Mathematical Analysis and Applications, 45(2): 432-454.

Ruan S and Wang W (2003). Dynamical behavior of an epidemic model with a nonlinear incidence rate. Journal of Differential Equations, 188(1): 135-163.

Song X and Cheng S (2005). A delay-differential equation model of HIV infection of CD4+ Tcells. Journal of the Korean Mathematical Society, 42(5): 1071-1086.

Wang X, Wei L and Zhang J (2014). Dynamical analysis and perturbation solution of an SEIR epidemic model. Applied Mathematics and Computation, 232:479-486.

Xiao D and Ruan S (2007). Global analysis of an epidemic model with non-monotonic incidence rate. Mathematicalk Biosciences, 208(2):419-429.

Yi N, Zhao Z and Zhang Q (2009). Bifurcations of an SEIQS epidemic model. International Journal of Information and Systems Sciences, 5(3-4): 296310.

Zhang J-Z, Jin Z, Liu Q-X and Zhang Z-Y (2008). Analysis of a Delayed SIR Model with Nonlinear Incidence Rate. Discrete Dynamics in Nature and Society, 2008, Article ID 636153, 16 pages. doi:10.1155/2008/636153. 